\title{
dc motion of ac driven sine-Gordon solitons
}

\author{
Niurka R. Quintero ${ }^{1}$, Angel Sánchez ${ }^{2}$ \\ Grupo Interdisciplinar de Sistemas Complicados, Departamento de Matemáticas, c/Butarque 15, Universidad Carlos III de Madrid, \\ E-28911 Leganés, Madrid, Spain
}

Received 1 December 1997; revised manuscript received 9 February 1998; accepted for publication 13 July 1998

Communicated by A.R. Bishop

\begin{abstract}
We investigate the dependence of dc soliton motion sustained by pure ac driving in the sine-Gordon model on various parameter problems, such as frequency, initial kink velocity or relative phase. We show by means of the collective coordinate formalism that ac driving induces a net dc velocity whose modulus and direction depend on the driving phase and on the kink initial velocity. Numerical simulations of the full sine-Gordon equation confirm the correctness and accuracy of this prediction. Nontrivial cases when dc soliton motion is transformed into oscillation as well as the effects of damping are analyzed. Our results generalize previous work on the undamped problem, and clarify a long standing issue about the existence and characteristics of this phenomenon in damped problems, whose possible appearance in other systems is also discussed.
\end{abstract}

PACS: $03.40 . \mathrm{Kf} ; 74.50 .+\mathrm{r} ; 85.25 . \mathrm{Cp}$

\section{Introduction}

It is well recognized that the study of nonlinear equations and their solutions is of great importance in many areas of physics. Among these, the sine-Gordon equation has attracted the interest of mathematicians and physicists due to not only its complete integrability but also its ubiquity as a model of nonlinear physical phenomena. Indeed, the sine-Gordon equation is known to be a canonical model for a wide variety of physical systems when topological solitons are present, such as motion of dislocations in crystals [1], charge density waves [2], solitons in magnetically ordered systems [3], epitaxial growth of thin films [4],

\footnotetext{
${ }^{1}$ E-mail address: niurka@dulcinea.uc3m.es.

${ }^{2}$ E-mail address: anxo@dulcinea.uc3m.es.
}

fluxon dynamics in long Josephson junctions [5], or DNA promoter dynamics [6].

One incompletely understood issue regarding the application of external forces on systems represented by the sine-Gordon model regards the effects of pure ac driving and the possibility that it induces dc motion of solitons. The corresponding perturbed sine-Gordon equation is

$$
\phi_{t t}-\phi_{x x}+\sin (\phi)=-\beta \phi_{t}+\epsilon f(t),
$$

where subindices indicate the derivative with respect to the corresponding variable, $\beta \phi_{t}$ is the usual damping term, and $f(t)=\sin \left(\delta t+\delta_{0}\right)$ is an external periodic force describing, for example, a long Josephson junction under the application of a uniform microwave field [7]. In the following, we will only consider ac driving (with or without dissipation) acting on a sin- 
gle soliton. We note that the influence of an ac force like the one we study here acting on breather solutions of the sine-Gordon equation was the subject of several papers by the end of the eighties, mostly from the view point of the use of a suitable frequency to stabilize breathers in the presence of dissipation $[8,9]$. The related problem of an ac driving in the presence of an additional dc driving has been already considered and it is quite well understood (see, e.g., Refs. $[7,10,11]$ and references therein), whereas the effects of pure dc driving were established in the seminal work of McLaughlin and Scott [12]. The issue of pure ac driving acting on solitons, however, has proven much more confusing, and a clear picture of soliton behavior under this kind of force was still lacking.

To our knowledge, work on this problem begins with the research by Olsen and Samuelsen in Ref. [13], where the authors studied the effect of $\mathrm{dc}$ and ac forces on sG kinks. In their analytical approach, they considered two special cases for an ac force given by $\epsilon \sin \left(\delta t+\delta_{0}\right)$, namely $\delta_{0}=0, \frac{1}{2} \pi$ with $\delta \neq 1$ and sufficiently small kink velocities. Their results were obtained in the approach that the solution of the perturbed $\mathrm{SG}$ equation can be divided into a kink part and a vacuum part. Although most of the paper deals with dc forces, the authors conclude that for the two cases studied for ac forces, one leads to kink dc motion $\left(\delta_{0}=0\right)$ whereas for the other $\left(\delta_{0}=\frac{1}{2} \pi\right)$ only oscillatory motion is found. For both choices, the work is further restricted to null initial velocity only, $u(0)=0$, with the subsequent loss of generality of their findings. Numerical simulations confirmed the predictions and the accuracy of the analytical calculations. However, no other initial phase differences were studied, and therefore a general analysis (including also initial velocity) was lacking. In addition, they did not present any results for ac driving plus damping, saying simply that similar results could be obtained in that case. Later and independently, Bonilla and Malomed studied this problem [14] (and subsequently in Ref. [15] for the Toda lattice with similar results), and claimed that ac driving could support de motion of solitons in a discrete (in space) sine-Gordon model, dissipation being crucial for this phenomenon. Unfortunately, as was shown in Ref. [16], this result was incorrect because of two reasons: First, it was obtained by means of a necessary, but not sufficient, existence condi- tion, and second, the authors used simultaneously and carelessly both the discrete and continuum limits of the model. Numerical simulations confirmed that the dc motion predicted in Ref. [14] did not take place [16]. Recently, another study found a parameter region for the damped discrete sine-Gordon model (i.e., the Frenkel-Kontorova chain) where ac driving can induce $\mathrm{dc}$ motion. Such behavior can only occur in the discrete model because its two ingredients arise from discreteness: the Peierls-Nabarro barrier and its associated frequency [17]. Hence this is a much more complicated (indeed, chaotic) process involying attractor competition and has nothing to do with the proposal of Ref. [14].

In this work, we show that dc motion of solitons induced by pure ac driving in the continuum sineGordon equation is indeed possible: In the next section, we use a collective coordinate approach to compute the soliton motion, and its mean velocity a function of the phase of the driving and the initial kink velocity. We verify our results by means of numerical simulation, the comparison turning out to be satisfactory with an almost perfect agreement. The final section contains a discussion of our results and comments on related subjects and generalizations to other systems.

\section{Collective coordinate analysis}

In order to perturbatively study Eq. (1) we will resort to the usual collective coordinate approach $[18,19]$ : If $\beta$ and $\epsilon$ are small, we can approximate the one-soliton solution of Eq. (1) by that of the unperturbed sine-Gordon equation $(\beta=\epsilon=0)$ with time dependent parameters, i.e., we will make the following ansatz,

$$
\begin{aligned}
& \phi(x, t)=4 \arctan \left[\exp \left( \pm \frac{x-x_{0}(t)-X_{0}(t)}{\sqrt{1-u^{2}(t)}}\right)\right] \\
& X_{0}(t)=\int_{0}^{t} u\left(t^{\prime}\right) \mathrm{d} t^{\prime}
\end{aligned}
$$

where the positive (negative) sign corresponds to a kink (anti-kink) solution. We note that $X(t)=$ $x_{0}(t)+X_{0}(t)(u(t))$ has the meaning of the position (velocity) of the center of the soliton, hence the name 
"collective coordinate," and that the main assumption underlying this approximation is that radiation effects induced by the perturbation are neglected. This will be verified a posteriori by comparing with the numerical simulations.

We now apply the method of McLaughlin and Scott [12] to obtain the equations of motion for the soliton center. The method, which they dubbed energetic analysis, is a rather simple one and amounts to computing the variation of the energy and momentum of the unperturbed sine-Gordon system due to the perturbation and the same quantity for a soliton solution, subsequently imposing compatibility. This is already a classic procedure and details can be found in Refs. $[12,18,19]$. The resulting equations of motion are (again, the \pm sign corresponds to a kink or an anti-kink)

$$
\begin{aligned}
& \frac{\mathrm{d} u}{\mathrm{~d} t}=-\frac{1}{4}\left(1-u^{2}\right)\left[ \pm \pi \sqrt{1-u^{2}} \epsilon f(t)+4 \beta u\right], \\
& \frac{\mathrm{d} X}{\mathrm{~d} t}=u(t) .
\end{aligned}
$$

We begin by discussing the dissipation-free case, i.e., we set $\beta=0$ in Eqs. (3). In this case, the first equation of (3) can be solved exactly, yielding

$$
\begin{gathered}
u(t)=\frac{F\left[u(0), \delta_{0}\right]}{\sqrt{1+F\left[u(0), \delta_{0}\right]^{2}}}, \\
F\left[u(0), \delta_{0}\right] \equiv \frac{u(0)}{\sqrt{1-u^{2}(0)}} \\
\pm \frac{\pi \epsilon}{4 \delta}\left[\cos \left(\delta t+\delta_{0}\right)-\cos \left(\delta_{0}\right)\right] .
\end{gathered}
$$

Eq. (4) should now be integrated to obtain the final result, namely the soliton center motion. This cannot be done in general; however, when $|u(0)| \ll 1$ and $|\pi \epsilon / 4 \delta| \ll 1$, a good approximation to the solution is given by

$$
\begin{gathered}
X(t)=X(0)+\left(u(0) \mp \frac{\pi \epsilon}{4 \delta} \cos \left(\delta_{0}\right)\right) t \\
\pm \frac{\pi \epsilon\left[\sin \left(\delta t+\delta_{0}\right)-\sin \left(\delta_{0}\right)\right]}{4 \delta^{2}} .
\end{gathered}
$$

From Eq. (5) it is evident that kinks and anti-kinks will move with net velocity $u(0) \mp\left[\pi \epsilon \cos \left(\delta_{0}\right)\right] / 4 \delta$ in a straight line, to which oscillations of frequency $\delta$ are overimposed. Therefore, in this approximation, an ac driving of frequency $\delta$ will almost always induce a dc motion of sine-Gordon solitons; indeed, only if

$u(0)= \pm \frac{\pi \epsilon \cos \left(\delta_{0}\right)}{4 \delta}$

will solitons remain oscillating around their initial position. It is important to note that this condition depends on the initial velocity of the soliton, $u(0)$; if $u(0)=0$, i.e., the soliton is initially at rest, the condition is simply $\delta_{0}=(2 n+1) \pi / 2, n=0, \pm 1, \ldots$ But even if the soliton is initially moving, a phase can be chosen such that this dc motion is stopped and transformed to an oscillation, a nontrivial and unexpected result.

We note that the above predictions have been obtained for the case of a slowly moving soliton and a small driving strength. It is possible, however, to establish this result in general by means of an alternative method based on the study of the Hamiltonian. This calculation is somewhat more complicated, so we will report on it elsewhere [20] and here we will just quote the final result, namely condition (6) for oscillatory motion becomes

$\frac{u(0)}{\sqrt{1-u(0)^{2}}}= \pm \frac{\pi \epsilon \cos \left(\delta_{0}\right)}{4 \delta}$

i.e., the only change with respect to the approximate condition previously derived is the (otherwise expected) appearance of the Lorentz factor $\gamma=$ $\sqrt{1-u(0)^{2}}$

To conclude our analytical calculations for this problem, we now turn to the dissipative case. Then, Eq. (3) cannot be solved, not even for $u$. A slow motion approximation $(|u(t)| \ll 1)$ yields

$$
\begin{aligned}
u(t) & =c \exp (-\beta t) \\
\mp & \frac{\pi \epsilon}{4\left(\beta^{2}+\delta^{2}\right)}\left[\beta \sin \left(\delta t+\delta_{0}\right)-\delta \cos \left(\delta t+\delta_{0}\right)\right],
\end{aligned}
$$

with

$$
\begin{aligned}
c= & u(0) \\
& \pm \frac{\epsilon \pi}{4\left(\beta^{2}+\delta^{2}\right)}\left[\beta \sin \left(\delta_{0}\right)-\delta \cos \left(\delta_{0}\right)\right]
\end{aligned}
$$

From the above, rather involved expression (or from the even more cumbersome one for $X(t)$ which can be obtained by integrating ( 8$)$ ), the main conclusion 
we can draw is that (within the small velocity approximation) solitons will never exhibit dc motion except for a transient, after which they will reach a final oscillatory state around a point depending on the initial conditions.

\section{Numerical results}

All the results and predictions in the previous section involve some approximation, beginning with the collective coordinate hypothesis of negligible radiation. Therefore, they are meaningless unless verified by numerical simulations of the full perturbed sineGordon problem (1). Simulations were carried out by means of a standard fourth order Runge-Kutta algorithm [21] with initial conditions given by an unperturbed sine-Gordon soliton, at rest or with velocity $u(0)$, and boundary conditions $\phi_{x}(L= \pm 50, t)=0$. The conclusion of our numerical simulation program was that the predictions of the collective coordinate theory are very well verified by the full perturbed sineGordon equation. An example of the accuracy of our analytical results is shown in Fig. 1, where we plot the results of simulations in the absence of dissipation for different values of the phase $\delta_{0}$ and a soliton (actually, a kink) initially at rest. The high degree of agreement between theory and simulations is apparent, confirming that dc motion of ac driven kinks is indeed the usual behavior, except for very particular choices of the phase of the driving.

The rest of the predictions are equally correct. Thus, Fig. 2 collects the outcome of simulations intended to verify the accuracy of the prediction that solitons with specific velocities can be stopped by a suitable choice of the driving phase. To verify this result, we proceed in the opposite way, i.e., we compute the critical velocity for a given value of the phase, and then we obtain the same value numerically. As can be seen from Fig. 2, the numerical value turns out to be $u(0)=0.1335$, to be compared to the predicted $u(0)=0.1347$, i.e., the accuracy is better than $1 \%$. Finally, Fig. 3 is an example of the behavior of the system when dissipation is present. We find numerically that, indeed, oscillatory motion is the only outcome of simulations for whatever value (not even small ones) of the initial velocity (which, in turn, agrees with the results in Ref. [16]).
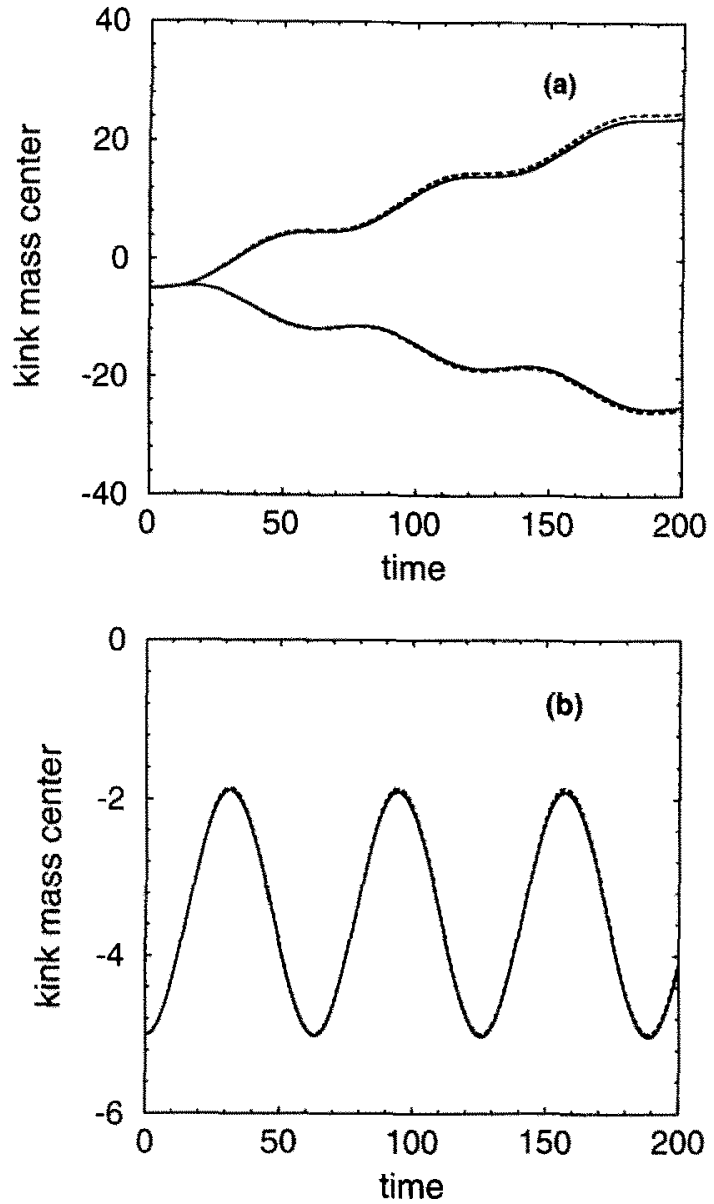

Fig. 1. Verification of collective coordinate predictions in the absence of dissipation. Simulation starts from a static kink located at $X(0)=-5$, and subject to an ac force given by $-0.02 \sin \left(0.1 t+\delta_{0}\right.$ ). (a) $\delta_{0}=0$ (upper curve), $\delta_{0}=\frac{3}{4} \pi$ (lower curve); notice that the direction of motion is opposite in both cases. (b) $\delta_{0}=\frac{1}{2} \pi$, critical value exhibiting oscillatory motion. Both in (a) and (b), solid lines correspond to numerical integration of $\mathrm{Eq}$. (1), dashed lines to the analytical prediction (5).

\section{Conclusions}

In summary, in this Letter we have analytically and numerically studied soliton dc motion induced by pure ac driving in sine-Gordon systems. The direction and modulus of the corresponding velocity have been shown to depend on the driving phase, which for a specific value (depending on the initial data) leads to pure ac motion even if the soliton was moving. The analytical results, obtained by means of a collective coordinate approach, agree with the numerical ones within an 


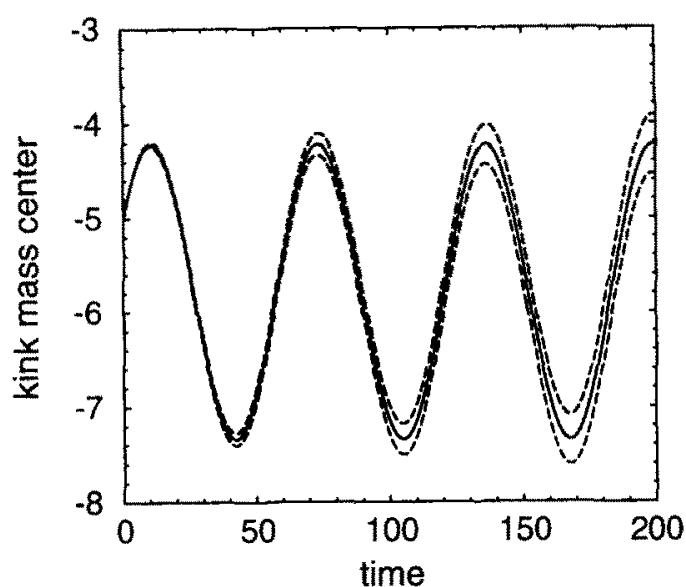

Fig. 2. Stopping of a kink initially moving with velocity $u(0)=0.1335$ (solid line) compared to the evolution of kinks moving with $u(0)=0.135$ (upper dashed curve) and $u(0)=0.132$ (lower dashed curve). The ac driving had $\delta_{0}=\frac{1}{6} \pi$; other parameters are $\delta=0.1$ and $\epsilon=0.02$. The theoretically predicted critical velocity was $u(0)=0.1347$, and the corresponding theoretical evolution lies on top of the numerical curve for $u(0)=0.1335$.

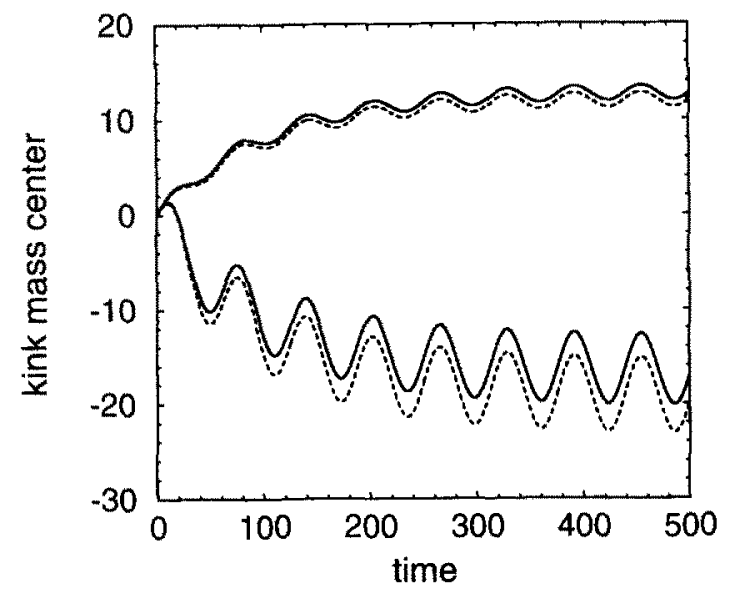

Fig. 3. Verification of collective coordinate predictions with dissipation. Shown are two examples of soliton motion subject to an ac force given by $\epsilon \sin \left(0.1 t+\delta_{0}\right)$, with $\epsilon=0.01$ (upper curve) and $\epsilon=0.05$ (lower curve); other parameters are $\beta=0.01, \delta_{0}=0.1$ and $u(0)=0.2$. Solid lines correspond to numerical integration of Eq. (1), dashed lines are the analytical predictions obtained by integrating $(8)$.

accuracy better than $1 \%$. We have also found that this effect is only possible in the absence of dissipation, and that the final steady state for all damping values is the oscillatory one. We note that the present work generalizes the results of Olsen and Samuelsen [13] for the nondissipative case, as their Eqs. (2.15)-(2.18) are particular cases of our results. In the nondissipative case, we clarify the assertion made by Olsen and Samuelsen [13], who said "similar (to the undamped case) results are obtained", not a very explicit statement. As we have seen, kink motion is never possible in this case, as shown also by Cai et al. by different arguments. This conclusion finally clarifies the problem of ac driving effects on solitons and opens the way to its applications in different technological contexts, such as, e.g., Josephson junctions [5,7].

To close this section, some comments are in order about the existence of this phenomenon in other systems. We believe that the features we have found here are generic in the collective coordinate sense, i.e., for systems whose solitons exhibit a clear and robust particle-like behavior (see, e.g., Refs. $[18,19]$ and references therein). However, care must be taken when dealing with solitons possessing inner degrees of freedom, such as the sine-Gordon breather or the $\phi^{4}$ solitary wave, as the frequencies pertaining to these degrees of freedom will necessarily interact in a complicated manner with the driving frequency. Indeed, Fernandez et al. [22] have shown that when an sG kink is placed on a harmonic potential well and is driven with a sinusoidal force with the frequency of the kink's natural oscillation, resonance phenomena appear, which supports our caveat regarding the interaction with internal modes. It is clear that this competition can give rise to chaotic phenomena, similar to that found in Ref. [17], overruling the simple scenario we have depicted here. On the other hand, if those frequencies are very different, our results should once again hold. Preliminary analytical and numerical investigations support this conclusion [20].

\section{Acknowledgement}

We thank Fernando Falo for discussions and for independent numerical checks of our predictions, and Esteban Moro and Franz Mertens for conversations on this work. Work at GISC (Leganés) has been supported by CICyT (Spain) grant MAT95-0325 and DGES (Spain) grant PB96-0119. 


\section{References}

(1) F.R.N. Nabarro, Theory of crystal dislocations (Dover, New York, 1987).

[2] G. Grüner, A. Zettl, Phys. Rep. 119 (1985) 117.

[3] A.M. Kosevich, B.A. Ivanov, A.S. Kovalev, Phys. Rep. 194 (1990) 117.

[4] J.D. Weeks, G.H. Gilmer, Adv. Chem. Phys. 40 (1979) 157.

[5] A. Barone, G. Paternó, Physics and applications of the Josephson effect (Wiley, New York, 1982).

[6] M. Salerno, Phys. Rev. A 44 (1991) 5292.

[7] M. Cirillo, P. Cocciolo, V. Merlo, N. Grønbech-Jensen, R.D. Parmentier, J. Appl. Phys. 75 (1994) 2125.

[8] P.S. Lomdahl, M.R. Samuelsen, Phys. Lett. A. 128 (1988) 427.

19] N. Gronbech-Jensen, Yu.S. Kivshar, M.R. Samuelsen, Phys. Rev. B 43 ( 1991 ) 5698.

[10] M. Cirillo, A.R. Bishop, N. Grønbech-Jensen, P.S. Lomdahl, Phys. Rev. E 49 ( 1994) R3606.
[11] L.M. Floria, F. Falo, Phys. Rev. Lett. 68 (1992) 2713.

[12] D.W. McLaughlin, A.C. Scott, Phys. Rev. A 18 (1978) 1652.

[13] O.H. Olsen, M.R. Samuelsen, Phys. Rev. B 28 (1983) 210.

[14] L.L. Bonilla, B.A. Malomed, Phys. Rev. B 43 (1991) 11539.

[15] B.A. Malomed, Phys. Rev. B 45 (1992) 4097.

[16] D. Cai, A. Sánchez, A.R. Bishop, F. Falo, L.M. Floría, Phys. Rev. B 50 (1994) 9652.

[17] P.J. Martínez, F, Falo, J.J. Mazo, L.M. Floria, A. Sánchez, unpublished (1997).

[18] Yu.S. Kivshar, B.A. Malomed, Rev. Mod. Phys. 61 (1989) 763.

[19] A. Sánchez, L. Vázquez, Int. J. Mod. Phys. B 5 (1991) 2825.

[20] N.R. Quintero, A. Sánchez, unpublished (1997).

[21] W.H. Press, S.A. Teukolsky, W.T. Vetterling, B.P. Flannery, Numerical Recipes in Fortran, 2nd Ed. (Cambridge Univ. Press, Cambridge, 1992).

[22] J.C. Fernández, M.J. Goupil, O. Legrand, G. Reinisch, Phys. Rev. B 34 (1986) 6207. 